\title{
Effets d'une partielle substitutionde la farine de poisson par la farine de soja sur la croissance des juvéniles de la perche du Nil (Lates niloticus, Linnaeus 1758)
}

\author{
Mouhamadou Amadou $\mathrm{LY}^{1,2^{*}}$ et Cheikh Tidiane BA ${ }^{2}$ \\ ${ }^{1}$ Universite Gaston Berger de Saint-Louis, Sénégal. \\ ${ }^{2}$ Universite Cheikh Anta Diop de Dakar, Sénégal. \\ *Auteur correspondant, E-mail : mamaly90@hotmail.com
}

\section{RESUME}

La présente étude a été menée pour évaluer les effets de l'incorporation de la farine de soja dans le régime alimentaire des juvéniles de la perche du Nil. Ce travail est réalisé sur des juvéniles de $1 \mathrm{~g}$ élevés dans des aquariums de 501 . Cinq régimes alimentaires isoprotéiques (45\% de protéines) contenant différents taux de farine de soja $0 \%, 26 \%, 36 \%, 51 \%$ et $60 \%$ noté respectivement $\mathrm{A}, \mathrm{B}, \mathrm{C}, \mathrm{D}$ et $\mathrm{E}$ ont été préparés. Chaque régime a été assigné de façon aléatoire à trois groupes de poissons nourris à satiété durant 8 semaines. Les résultats obtenus montrent que les meilleures performances de croissance et d'efficacité alimentaire sont obtenues avec les régimes nourris avec la farine de poisson (A), suivis dans l'ordre des régimes $\mathrm{B}, \mathrm{C}, \mathrm{D}$ et $\mathrm{E}$. Les taux de croissance spécifiques (TCS) varient entre $3,26 \%$ et $3,77 \%$ respectivement chez les poissons nourris avec les régimes $\mathrm{A}, \mathrm{B}, \mathrm{C}, \mathrm{D}$ et $\mathrm{E}$. Le coefficient d'efficacité protéique (CEP) a varié de 2,07 à 1,6. Les résultats statistiques montrent que le meilleur $\mathrm{CEP}$ est obtenu avec le régime $\mathrm{A}(\mathrm{P}<0.05)$ et le plus faible avec le régime $\mathrm{E}$. Les résultats de la présente étude indiquent qu'il est possible d'incorporer $51 \%$ de la farine de soja dans l'alimentation des juvéniles de Lates niloticus sans compromettre leur croissance.

(C) 2015 International Formulae Group. All rights reserved.

Mots clés: Perche du Nil, Lates niloticus, farine de soja, grossissement, alimentation.

\section{INTRODUCTION}

La perche du Nil, Lates niloticus (Linnaeus, 1758), appartenant à la famille des Centropomidae, est un carnivore d'eau douce repartie dans la région éthiopienne de l'Afrique, se produisant généralement dans tous les grands bassins fluviaux, dont le Nil, le Tchad, le Sénégal, la Volta et du Congo. La perche du Nil est menacée d'extinction due à une surexploitation, (Munyaho, 2004; Njiru et al., 2009). Le développement de l'élevage de cette espèce dépendra principalement de la disponibilité des juvéniles de qualité et le développement d'un aliment équilibrée et adaptée. Les besoins nutritionnels des juvéniles de perche du Nil sont encore mal connus. Des travaux réalisés précédemment ont rapporté que les juvéniles de Lates niloticus ont un besoin de $45 \%$ de protéines (Ly, 2014). Le coût de l'aliment représente $60-70 \%$ du coût de la production piscicole intensive. Toute réduction de la teneur en protéines des régimes sans affecter la croissance du poisson peut réduire sensiblement le coût de l'alimentation chez les poissons (Kim et al., 2003). La farine de 
poisson est très utilisée dans l'alimentation des poissons en raison de sa richesse en acides aminés essentiels et de sa palatabilité. Cependant, la demande croissante, le coût élevé et l'instabilité de l'approvisionnement de farine de poisson ont orienté les recherches vers des sources alternatives de protéines, notamment les protéines végétales (Kenan et Chris, 2005). Parmi les protéines végétales, le tourteau de soja est l'ingrédient le plus utilisé en raison de sa disponibilité, de son prix comparé au prix de la farine de poisson et de sa bonne valeur nutritionnelle (Hernandez et al., 2004 ; Rondan et al., 2004). La recherche sur la substitution partielle de la farine de poisson par la farine de soja sur la croissance des juvéniles de la perche du Nil, est une contribution pour le développement d'un aliment économique pour cette espèce. Des essais sur la substitution partielle de la farine de poisson par la farine de soja sur la croissance ont donc été effectués, pour le développement d'un aliment économique des juvéniles de la perche du Nil.

\section{MATERIEL ET METHODES Procédure expérimentale}

Au début de l'essai d'alimentation des juvéniles de la perche du Nil d'eau douce sauvage, Lates niloticus, pesant environ $1.01 \mathrm{~g}$ ont été utilisés dans cette étude. Ces poissons ont été obtenus grâce à l'aide d'un pêcheur dans la localité du barrage de Diama, à SaintLouis, Sénégal. Les poissons ont été stockés dans des aquariums deux semaines avant le début de l'expérience pour les acclimater aux conditions expérimentales. Pendant cette période, ils ont été nourris avec un aliment commercial de clarias obtenu à l'écloserie de l'Agence nationale de l'aquaculture situé dans le district de Richard-Toll.

Au début de l'expérience, les poissons ont été pesés et comptés. Chaque régime expérimentale a été assigné, de façon aléatoire, à 3 groupes de 15 poissons (poids moyen : $1,29 \pm 0,02 \mathrm{~g}$ ) par aquarium. Chaque aquarium a été constamment aérer et son niveau d'eau maintenu à 501 . Chaque régime est nourri à satiété visuelle, 3 fois par jour à 08h:00 ; 12h: 00 et 17h:00 sur une période de 30 min pendant 8 semaines.

Les poissons ont été soumis à une photopériode de $12 \mathrm{~h}$ nuit et de $12 \mathrm{~h}$ jour et tous les aquariums eurent des conditions identiques d'éclairage. Les taux d'oxygène dissous et de la température de l'eau ont été relevés tous les jours et leur moyenne respectives ont été $6.5 \mathrm{mg} / 1$ et $30{ }^{\circ} \mathrm{C}$. Les poissons de chaque aquarium étaient péses toutes les 2 semaines, les poissons étaient privés de nourriture $24 \mathrm{~h}$ avant et $24 \mathrm{~h}$ après les opérations de pesées. Les aquariums étaient nettoyés tous les jours le matin pour réduire le bio-film et éliminer certains déchets accumulés. Les granules non consommés ont été collectés 30 minutes après chaque repas pour estimer la consommation alimentaire, tandis que les particules dissoutes ont été négligées dans cette étude.

\begin{abstract}
Aliment expérimental
Cinq régimes ont été formulés pour contenir différents teneur de farine de soja en remplacement de la farine de poisson. Tous les régimes ont $45 \%$ de protéine. Le régime $\mathrm{A}$ servant de control contient $78 \%$ de farine de poisson et $0 \%$ de farine de soja. Les régimes B, C, D et E contiennent $26 \%, 36 \%, 51 \%$ et $60 \%$ de farine de soja et $57 \%, 48 \%, 35 \%$ et $27 \%$ de farine de poisson respectivement. Les proportions de farines de poisson et de soja ont été choisi pour que les régimes soit isoprotéiques. Les vitamines, sels minéraux et liant sont restés identiques d'un régime à l'autre pour éviter d'influencer les résultats. Les farines de poissons et de soja ont été finement broyées et tamisées à travers des mailles de125 $\mu \mathrm{m}$. Les mixtures de vitamines et minéraux; la cellulose et le liant ont été mélangés séparément avant d'être ajouté au mélange principal. De surcroît de l'huile de poisson et de soja ont été ajoutés au mélange avant l'addition de l'eau (Tableau 1). Le mélange semi-humide est versé, puis pressé dans un hachoir à viande, à l'aide d'une maille de $2 \mathrm{~mm}$, donnant des filaments en forme de spaghettis. Ces derniers sont séchés au soleil pendant deux jours, coupés aux dimensions
\end{abstract}


souhaitées, ensachés et stockés dans un congélateur jusqu'à son utilisation. Les granules sont tamisés avant l'alimentation pour enlever les petites particules.

\section{Echantillonnage et analyses biochimiques}

Les analyses biochimiques (protéines, lipides, teneur en eau et cendres) ont été effectuées en triplicata selon les méthodes standards de l'AOAC (1990) et ont concerné les aliments expérimentaux, les carcasses homogénéisées de 10 poissons prélevés en début d'expérience et les carcasses homogénéisées de 3 poissons prélevés aléatoirement en fin d'expérience dans chacun des aquariums expérimentaux.

Les lipides ont été estimés par la méthode d'extraction d'éther (Soxtec système HT6; Tecator). Les protéines brutes ont été déterminées $(\mathrm{N} \times 6.25)$ par la méthode de Kjeltec 1002 (Tecator) après une digestion acide. La cellulose brute est analysée par la méthode fibertec M1020 (Foss Tecator). Les cendres ont été déterminées après incinération des échantillons au four à moufle à $550{ }^{\circ} \mathrm{C}$ pendant 12 heures et la matière sèche est déterminée par mesure de la perte de poids après séchage durant $24 \mathrm{~h}$ à l'étuve à $105^{\circ} \mathrm{C}$. Plusieurs paramètres ont été régulièrement contrôlés pour s'assurer de la bonne qualité de l'eau. La température de l'eau et l'oxygène dissous ont été mesurée successivement tous les jours à l'aide d'un appareil YSIModèle58 (Yellow Springs Instrument, Yellow Springs, Ohio, USA). Le pH de l'eau a été mesuré tous les jours par un testeur de $\mathrm{pH}$ de la série DMT-30.

\section{Calcule et analyses statistiques}

Des résultats obtenus, plusieurs paramètres ont été calculés pour évaluer les performances zootechniques. Ces paramètres sont le coefficient d'efficacité protéique (CEP) exprimé en pourcentage, le taux de croissance spécifique (TCS), le taux de conversion alimentaire (TCA), et le taux de survie (TS). Les formules de calcul de ces paramètres sont :
- $\quad$ le coefficient d'efficacité protéique = Biomasse produite / Protéine consommé ;

- le taux de croissance spécifique $(\mathrm{TCS}, \% /$ jour $)=100 *(($ Ln Poids final - Ln Poids initial) / durée) ;

- le taux de conversion alimentaire = (Quantité d'aliment ingéré rapportée à la matière sèche)/ (Biomasse produite);

le taux de survie $(\%)=100 *$ (nombre de poissons à la fin de l'expérience / nombre de poissons au début de l'expérience).

Les résultats ont été présentés sous forme de moyenne $\pm \mathrm{ESM}$. Les résultats sont comparés statistiquement par l'analyse de la variance à un critère (ANOVA) pour tester l'effet de 5 formules alimentaires et ceci après vérification préalable de l'homogénéité des variances et de la normalité des données à analyser. Les effets du traitement ont été considérées comme significatives à un seuil de $5 \% \quad(\mathrm{P}<0,05)$. Lorsque l'analyse de variance se révélait significatif, le test du Waller-Duncan a été utilisé pour la comparaison pariée des moyennes. Toutes les analyses statistique ont été réalisées à l'aide du logiciel de statistique SAS / PC (SAS Institute Inc).

\section{RESULTATS}

$\mathrm{Au}$ cours de l'expérimentation, la température de l'eau a varié de 29 à $30{ }^{\circ} \mathrm{C}$, la concentration en oxygène dissous de 5,5 à $7 \mathrm{mg} / \mathrm{let}$ le $\mathrm{pH}$ de 7,5 à 8 .

Les résultats des paramètres zootechniques chez Lates niloticus au cours de l'expérience sont consignés dans le Tableau 2. Les meilleures performances de croissance et d'efficacité alimentaire sont obtenues avec les régimes nourris avec la farine de poisson (A), suivis dans l'ordre des régimes $\mathrm{B}, \mathrm{C}, \mathrm{D}$ et $\mathrm{E}$. Les résultats obtenus montrent que les taux de croissance spécifiques (TCS) varient entre $3.26 \%$ et $3.77 \%$ respectivement chez les poissons nourris avec les régimes A, B, C, D et E. Il y'a eu une différence significative entre les régimes alimentaires $(\mathrm{P}<0.05)$. Le coefficient d'efficacité protéique (CEP) a varié de 2.07 à 1.6. Les résultats statistiques montrent que le 
meilleur CEP est obtenu avec le régime $\mathrm{A}$ $(\mathrm{P}<0.05)$ et le plus faible avec le régime $\mathrm{E}$. Pour les lots de poisson recevant les régimes $\mathrm{A}, \mathrm{B}, \mathrm{C}, \mathrm{D}$ et $\mathrm{E}$, le taux de conversion alimentaire (TCA) est compris entre 1.07 et $1.40 \mathrm{~kg}$ de nourriture sèche par $\mathrm{kg}$ de poids frais produit. Le lot de poisson nourri avec le régime $\mathrm{E}$ a obtenu le TCA le plus élevé $(\mathrm{P}<$ 0.05). Le meilleur TCA est obtenu avec le régime $\mathrm{A}$ comparé aux autres régimes $\mathrm{C}, \mathrm{D}$ et $\mathrm{E}$; tandis que la différence entre le TCA des régimes $\mathrm{A}$ et $\mathrm{B}$ n'est pas significative $(\mathrm{P}>0.05)$. Pour ce qui concerne les taux de survie, les analyses statistiques montrent que seul le lot nourri avec le régime $\mathrm{E}$ diffère significativement $(\mathrm{P}<0.05)$ des autres lots.

Les résultats des analyses biochimiques des poissons nourris avec les cinq régimes alimentaires sont consignés dans le Tableau 3. Les teneurs en eau des poissons en fin d'expérience ont diminué avec tous les régimes comparativement à l'état initial. Les poissons nourris avec le régime alimentaire $\mathrm{E}$ ont été plus riche en eau $(\mathrm{P}<0.05)$ comparés à ceux nourris avec les régimes $\mathrm{A}$ et $\mathrm{B}$. Tandis que pour les régimes $\mathrm{C}, \mathrm{D}$ et $\mathrm{E}$ aucune différence significative entre les régimes n'a été observé $(\mathrm{P}>0.05)$. Les résultats montrent que les teneurs en lipides corporelles des poissons décroissent avec l'augmentation de la quantité de la farine de soja incorporée dans le régime alimentaire. Tous les lots de poisson sont plus gras en fin qu'en début d'expérience (Tableau 3). Les résultats statistiques montrent que les poissons du lot E présentent la teneur la plus faible $(\mathrm{P}<0.05)$ comparés à ceux nourris avec les régimes A, B, C et D. En ce qui concerne la teneur en protéines corporelles, l'analyse statistique montre qu'elle augmente de façon significative avec le taux d'incorporation de la farine de poisson dans le régime alimentaire. Les lots de poisson nourris avec le régime A présentent la teneur la plus élevé, suivis par les poissons nourris avec les régimes $\mathrm{B}, \mathrm{C}$ et $\mathrm{D}$, tandis que les valeurs les plus faibles sont obtenues avec les poissons nourris avec le régime $\mathrm{E}(\mathrm{P}<$ $0.05)$.

Tableau 1: Formulation et composition des régimes alimentaires testés de la perche de Nil (Lates niloticus).

\begin{tabular}{|c|c|c|c|c|c|}
\hline \multirow[b]{2}{*}{ Ingrédient (g/100g) } & \multicolumn{5}{|c|}{ Régimes alimentaires } \\
\hline & $\mathbf{A}$ & B & $\mathbf{C}$ & D & $\mathbf{E}$ \\
\hline Farine de poisson & 78 & 57 & 48 & 35 & 27 \\
\hline Farine de soja & 0 & 26 & 36 & 51 & 60 \\
\hline Cellulose & 10 & 5 & 4 & 3 & 6 \\
\hline Huile de poisson : huile de soja $(7: 3)$ & 2 & 4 & 5 & 5 & 1 \\
\hline CMV & 4 & 4 & 4 & 4 & 4 \\
\hline Liant & 6 & 4 & 3 & 2 & 2 \\
\hline \multicolumn{6}{|l|}{ Composition biochimique (\%MS) } \\
\hline Matières sèche & 87,8 & 88,02 & 88,37 & 89,27 & 90,93 \\
\hline Protéines & 45,47 & 45,87 & 45,48 & 45,19 & 44,9 \\
\hline Lipides & 9,9 & 10,2 & 10,48 & 9,47 & 9,84 \\
\hline Fibres & 3,80 & 4,02 & 4,61 & 7,56 & 7,64 \\
\hline Cendres & 15,43 & 13,61 & 12,72 & 11,48 & 10,13 \\
\hline $\mathrm{ENA}^{\mathrm{a}}$ & 13,20 & 14,32 & 15,07 & 15,56 & 18,42 \\
\hline
\end{tabular}


Tableau 2. Performances zootechniques des différents lots de la perche du Nil (Lates niloticus) nourris avec cinq régimes alimentaires durant 8 semaines d'experimentation ${ }^{1}$.

\begin{tabular}{lccccc}
\hline \multirow{2}{*}{ Variables } & \multicolumn{5}{c}{ Régimes alimentaires } \\
\cline { 2 - 6 } & $\mathbf{A}$ & $\mathbf{B}$ & $\mathbf{C}$ & $\mathbf{D}$ & $\mathbf{E}$ \\
\hline CEP & $2.07 \pm 0.12^{\mathrm{a}}$ & $1.87 \pm 0.10^{\mathrm{b}}$ & $1.83 \pm 0.00^{\mathrm{b}}$ & $1.75 \pm 0.08^{\mathrm{bc}}$ & $1.60 \pm 0.11^{\mathrm{c}}$ \\
TCS & $3.77 \pm 0.02^{\mathrm{a}}$ & $3.59 \pm 0.07^{\mathrm{b}}$ & $3.45 \pm 0.03^{\mathrm{c}}$ & $3.44 \pm 0.02^{\mathrm{c}}$ & $3.26 \pm 0.02^{\mathrm{d}}$ \\
TCA & $1.07 \pm 0.06^{\mathrm{c}}$ & $1.17 \pm 0.06^{\mathrm{bc}}$ & $1.20 \pm 0.0^{\mathrm{b}}$ & $1.27 \pm 0.06^{\mathrm{b}}$ & $1.40 \pm 0.10^{\mathrm{a}}$ \\
TS $(\%)$ & $100^{\mathrm{a}}$ & $100^{\mathrm{a}}$ & $100^{\mathrm{a}}$ & $100^{\mathrm{a}}$ & $95.55 \pm 3.85^{\mathrm{b}}$ \\
\hline${ }^{ }$Les résultants sont exprimés en : Moyenne \pm EMS de trois répétitions $(\mathrm{n}=3)$. Sur chaque ligne, les moyennes \pm \\
EMS, affectées par des lettres différentes, sont significativement différentes $(\mathrm{P}<0.05)$.
\end{tabular}

Tableau 3. Composition biochimique de la carcasse des poisons en début et fin d'expérience des juvéniles de la perche du Nil ${ }^{1}$.

\begin{tabular}{lcccccc}
\hline \multirow{2}{*}{$\begin{array}{l}\text { Composition } \\
(\%)\end{array}$} & \multirow{2}{*}{ Etat initial } & \multicolumn{5}{c}{ Etat final (Régimes alimentaires) } \\
\cline { 3 - 7 } & & $\mathbf{A}$ & $\mathbf{B}$ & $\mathbf{C}$ & $\mathbf{D}$ & $\mathbf{E}$ \\
\hline Teneur en eau & $78.11 \pm 0.11$ & $74.09 \pm 0.06^{\mathrm{a}}$ & $74.15 \pm 0.05^{\mathrm{a}}$ & $74.34 \pm 0.13^{\mathrm{ab}}$ & $74.57 \pm 0.22^{\mathrm{b}}$ & $74.60 \pm 0.06^{\mathrm{b}}$ \\
Protéines & $52.30 \pm 0.61$ & $54.09 \pm 0.02^{\mathrm{a}}$ & $53.62 \pm 0.20^{\mathrm{b}}$ & $53.39 \pm 0.20^{\mathrm{b}}$ & $53.21 \pm 0.39^{\mathrm{b}}$ & $52.57 \pm 0.19^{\mathrm{c}}$ \\
Lipides & $10.75 \pm 0.09$ & $12.69 \pm 0.02^{\mathrm{a}}$ & $12.63 \pm 0.12^{\mathrm{a}}$ & $12.37 \pm 0.02^{\mathrm{b}}$ & $12.27 \pm 0.01^{\mathrm{b}}$ & $11.17 \pm 0.01^{\mathrm{c}}$ \\
Cendres & $20.18 \pm 0.17$ & $20.25 \pm 0.07^{\mathrm{a}}$ & $20.27 \pm 0.03^{\mathrm{b}}$ & $20.36 \pm 0.02^{\mathrm{bc}}$ & $20.33 \pm 0.09^{\mathrm{bc}}$ & $20.74 \pm 0.08^{\mathrm{c}}$ \\
\hline
\end{tabular}

Les résultants sont exprimés en : Moyenne \pm EMS de deux répétitions $(\mathrm{n}=3)$. Sur chaque ligne, les moyennes \pm EMS, affectées par des lettres différentes, sont significativement différentes $(\mathrm{P}<0.05)$. L'absence de lettre sur une même ligne indique une absence de différence significative $(\mathrm{P}>0.05)$.

\section{DISCUSSION}

De nombreuses études ont été réalisées pour étudier l'utilisation de la farine de soja dans les régimes alimentaires pour les poissons telles que Heterotis niloticus (Monentcham et al., 2010), Sparus aurata (Nengasetal., 2008), et Dicentrqrchus labrax (Tibaldi et al., 2006).

La présente étude est la première à notre connaissance concernant la substitution de la farine de poisson par la farine de soja chez des juvéniles de la perche du Nil. Les résultats obtenus montrent qu'il est possible d'incorporer la farine de soja jusqu'à $51 \%$ dans un régime alimentaire pour le grossissement de la perche du Nil. En effet, l'inclusion de la farine de soja jusqu'à $51 \%$ affecte légèrement les performances zootechniques chez la perche du Nil. Au-delà de ce seuil, une réduction nette de la croissance et une mauvaise transformation alimentaire est observée. Des résultats similaires sur des études effectuées sur d'autres espèces ont été observés par Schaeffer et al. (2011) qui indiquent qu'il est possible d'inclure jusqu'à $49.5 \%$ de la farine de soja sans effet négatif sur la croissance de la perche jaune. Pour Kasper et al. (2007), il est possible d'incorporer sans conséquences négatives un seuil de $50 \%$ de farine de soja sur la croissance des juvéniles de la perche jaune.

Selon Monentcham et al. (2010), chez Heterotis niloticus, le remplacement de la farine de poisson par une mixture des farines soja et de la graine de coton à concurrence de $50 \%$ est sans effet négatif sur sa croissance. Tandis que l'étude de Rodiles et al. (2012) sur la Sole sénégalaise établit que $30 \%$ de la farine de poisson peut être remplacé par des dérivés de soja sans incidence ni sur la croissance, ni sur les protéases intestinales de cette espèce.

La mauvaise croissance observée dans le régime E peut être liée à la présence des facteurs antinutritionnels présents dans la farine de soja. La présence de facteurs antinutritionnels dans les matières d'origines végétales affecte directement (inhibiteurs de trypsine, acides phytiques qui complexe le 
phosphore et le rend indispensable) ou indirectement (oligosaccharides, tannins, glucosinolates dont les métabolites affectent l'appétit et perturbent la synthèse des hormones thyroïdiennes), la capacité digestive des poissons (Pham et al., 2007).

Le faible taux du coefficient d'efficacité protéique au-delà d'une incorporation de $50 \%$ de farine de soja peutêtre dû à une déficience en acides aminés essentiels dans la farine de soja. Médale et Kaushik (2009) ont rapporté que quand l'apport alimentaire en acides aminés ne répond pas parfaitement aux besoins de l'animal, le catabolisme azoté augmente, la rétention protéique est réduite et donc la croissance est ralentie et les rejets azotés accrus.

La substitution de la farine de poisson par des produits végétaux provoque des modifications des marqueurs du système immunitaire au niveau du foie et de l'intestin chez toutes les espèces de poisson (SitjàBobadilla et al., 2005; Tacchi et al., 2012).

L'analyse biochimique de l'aliment montre que le régime $\mathrm{E}$ est le plus riche en extractif non azoté (ENA) d'où riche en glucides complexes. La forte teneur en fibre de l'aliment E peut expliquer les mauvaises performances de la perche du nil nourris avec ce régime. Les fibres accélèrent le transit gastro-intestinal; d'où une faible efficacité digestive des enzymes et par conséquent une diminution de l'absorption des nutriments (Médale et al., 2013). De plus, il a été observé que les fibres peuvent réduire la biodisponibilité des nutriments (Médale et al., 2013).

En général, une faible croissance est observée chez le poisson nourris avec des protéines d'origine végétale, ceci est due à la faible appétibilité, déficiences en certains acides aminés, faible teneur en phosphore, forte teneur en extractif non azoté et enfin un trouble de métabolisme lipidique (Gatlin III et al., 2007 : Nengas et al., 2008 ; Médale et al., 2013). La synthèse protéique et la croissance des poissons sont affectés par la déficience d'un ou de plusieurs acides aminés dans l'aliment. Gatlin III et al. (2007) ont rapporté que les faibles teneurs en lysine et méthionine dans la farine de soja représentent une limite de leur utilisation dans les aliments pour poisson. Il a été observé une réduction de la croissance chez la daurade nourrie avec un régime de $30 \%$ de remplacement de la farine de poisson par la farine de soja, (Nengas et al., 2008)

L'analyse de la composition biochimique de la carcasse des poissons a montré que les teneurs en lipides corporelles des poissons décroissent avec l'augmentation de la quantité de la farine de soja incorporée dans le régime alimentaire. Des résultats similaires ont été observés par Azaza et al. (2006) chez le tilapia et par Siddhuraju et Becker (2001) chez la Carpe commune nourrie avec des régimes contenant des matières premières d'origine végétales a des taux élevés. Par contre, Tibaldi et al. (2006) ont rapporté que l'inclusion de différents types et taux de farine de soja n'ont pas eu d'influence sur la composition biochimique de la carcasse chez le bar Européen. Les mortalités observés sont dues au refus de deux sujets, qui se suffisaient à 2 ou 3 graines d'où leur affaiblissement et finalement leur périssement.

\section{Conclusion}

La recherche sur la substitution partielle de la farine de poisson par la farine de soja sur la croissance des juvéniles de la perche du Nil, est une contribution pour le développement d'un aliment économique pour cette espèce. En conclusion, les résultats de la présente étude indiquent qu'il est possible d'incorporer $51 \%$ de la farine de soja dans l'alimentation des juvéniles de Lates niloticus sans compromettre leur croissance.

\section{REFERENCES}

AOAC. 1990.Official Methods of Analysis of the Association of Official Analytical Chemists (15th edn). AOAC: Arlington, Virgina, USA.

Azaza MS, Mensi F, Toko II, Dhraief MN, Abdelmouleh BB, Kraiem MM. 2006. 
Effets de l'incorporation de la farine de tomate dans l'alimentation du tilapia du nil (Oreochromis niloticus, L., 1758) en élevage dans les eaux géothermales du sud Tunisien. Bull. Inst. Natn. Scien. Tech. Mer de Salammbo., 33: 47-58.

Gatlin DM III, Barrows FT, Brown P, Dabrowski K, Gaylord TG, Hardy RW, Herman E, Hu GS, Krogdahl A, Nelson R, Overturf K, Rust M, Sealey W, Skonberg D, Souza EJ, Stone D, Wilson R, Wurtele E. 2007. Expanding the utilization of sustainable plant productsin aquafeeds: a review. Aquac Res., 38: 551-579

Hernandez C, Sarmiento-Pardo J, GonzalezRodrigez B, Isabel AP. 2004. Replacement of fish meal with coextruded wet tuna viscera and corn meal in diets for white shrimp (Litopenaeus vannamei Boone), Aquaculture Research, 35(12): 1153-1157.

Kasper CS, Watkins BA, Brown PB. 2007. Evaluation of two soybean meals fed to yellow perch (Perca flavescens), Aquaculture Nutrition, 13: 431-438.

Kenan E, Chris GC. 2005. Fish meal replacement by plant and animal byproducts in diets for the Australian shortfinned eel, Anguilla australis australis (Richardson), Aquaculture Research, 36(5): 445-454.

Kim K, Wang X, Bai SC. 2003. Reevaluation of the dietary protein requirement of Japanese flounder (Parlichthys olivaceus). J. World. Aquacult. Soc., 34(2): 133-139.

Ly MA. 2014. Effect of Dietary Protein Level on Growth and Body Composition of Juveniles Nile Perch (Lates niloticus, Linnaeus 1758). Journal of Biology and Life Science, 5(1):31-40.

Médale F, Kaushik S. 2009. Les sources protéiques dans les aliments pour les poissons d'élevage. Cah Agric., 18: 103111.

Médale F, Le BR, Dupond NM, Quillet E, Aubin J, Panserat S. 2013. Des aliments à base de végétaux pour les poissons d'élevage. INRA Prod. Anim., 26(4): 303-316.

Monentcham SE, Pouomogne V, Kestemont P. 2010. Influence of dietary protein levels on growth performance and body composition of African fingerlings, Heterotis niloticus (Cuvier, 1829). Aquacult. Nutr., 16: 144-162.

Munyaho AT. 2004. Assesment of the status of the stock and fishery of Nile perch in Lake Victoria, Uganda. Final Project, Fisheries Training Programme. The United Nations University, Iceland. Nengas I, Alexis MN, Davies SJ. 2008. Partial substitution of fishmeal with soybean meal products and derivatives in diets for the gilthead sea bream (Sparus aurata L.). Aquaculture Research., 27: 147-156.

Njiru M, Getabu A, Taabu AM, Mlaponi E, Muhoozi L, Mkumbo OC. 2009. Managing Nile perch using slot size: is it possible? Afr. J. Trop. Hydrobiol. Fish., 12:9-14.

Pham MA, Lee KJ, Lim SJ, Park KH. 2007. Evaluation of cottonseed and soybean meal as partial replacement for fishmeal in diets for juvenile Japanese olive flounder (Paralichthys olivaceus). Fisheries Science, 73: 760-769.

Rodiles A, Santigosa E, Herrera M, HacheroCruzado I, Cordero ML, MartínezLlorens S, Lall SP. 2012.Effect of dietary protein level and source on digestive proteolytic enzyme activity in juvenile Senegalese sole, Solea senegalensis Kaup 1850. Aquaculture International., 20: 1053-1070.

Rondan M, Hernandez DM, Egea A, Garcia B, Jover M, Rueda FM, Martinez JF. 2004. Effects of fishmeal replacement with soybean meal as protein source, and protein replacement with carbohydrates as an alternative energy source on sharpsnout sea bream, Diplodus puntazzo, and fatty acid profile, Aquaculture Research, 35(13): 12201227. 
Schaeffer TW, Brown ML, Rosentrater KA. 2011. Effects of Dietary Distillers Dried Grains with Solubles and Soybean Meal on Extruded Pellet Characteristics and Growth Responses of Juvenile Yellow Perch. North American Journal of Aquaculture, 73: 270-278.

Siddhuraju P, Becker K. 2001. Preliminary nutritional evaluation of mucuna seed meal (Mucuna pruriens varutilis) in common carp (Cyprinus carpio L.): an assessment by growth performance and feed utilization. Aquaculture, 196: 105123.

Sitjà-Bobadilla, A, Peña-Llopis S, GómezRequeni P, Médale F, Kaushik S, PérezSánchez, J. 2005. Effect of fish meal replacement by plant protein sources on non-specific defence mechanisms and oxidative stress in gilthead sea bream
(Sparus aurata). Aquaculture, 249: 387400.

Tacchi L, Secombes CJ, Bickerdike R, Adler MA, Venegas C, Takle H, Martin SAM. 2012. Transcriptomic and physiological responses to fishmeal substitution with plant proteins in formulated feed in farmed Atlantic salmon (Salmo salar). BMC Genomics, 13: 1-21.

Tibaldi E, Hakim Y, Uni Z, Tulli F, Francesco M, Luzzana U, Harpaz S. 2006. Effects of the partial substitution of dietary fish meal by differently processed soybean meals on growth performance, nutrient digestibility and activity of intestinal brush border enzymes in the European sea bass (Dicentrarchus labrax). Aquaculture, 261: 182-193. 